\title{
En-bloc resection of urinary bladder tumour - a prospective controlled multicentre observational study
}

\author{
Sławomir Poletajew ${ }^{1}$, Wojciech Krajewski ${ }^{2}$, Paweł Stelmach $^{3}$, Jan Adamowicz ${ }^{4}$, Łukasz Nowak ${ }^{2}$, Marco Moschini $^{5}$, \\ Piotr Zapała ${ }^{6}$, Tomasz Drewa ${ }^{4}$, Andrzej Paradysz ${ }^{3}$, Piotr Radziszewski ${ }^{6}$, Romuald Zdrojowy ${ }^{2}$, Piotr Kryst ${ }^{1}$ \\ ${ }^{1}$ Second Department of Urology, Centre of Postgraduate Medical Education, Warsaw, Poland \\ ${ }^{2}$ Department of Urology and Oncological Urology, Wroclaw Medical University, Wroclaw, Poland \\ ${ }^{3}$ Department of Urology, School of Medicine with the Division of Dentistry in Zabrze, Medical University of Silesia in Katowice, Poland \\ ${ }^{4}$ Department of Regenerative Medicine, Collegium Medicum in Bydgoszcz, Nicolaus Copernicus University in Torun, Poland \\ ${ }^{5}$ Department of Urology, Luzerner Kantonsspital, Luzern, Switzerland \\ ${ }^{6}$ Department of General, Oncological and Functional Urology, Medical University of Warsaw, Warsaw, Poland
}

Videosurgery Miniinv 2021; 16 (1): 145-150 DOI: https://doi.org/10.5114/wiitm.2020.95399

\begin{abstract}
Introduction: Transurethral resection of bladder tumour (TURBT) is one of the most commonly performed urologic procedures. Because of the shortcomings of conventional TURBT, the en-bloc resection concept was created.

Aim: To analyse the influence of en-bloc technique on surgical and oncological outcomes of TURBT performed with electric current.

Material and methods: This non-randomized, prospective controlled multicentre study enrolled 427 consecutive patients undergoing TURBT performed by five experienced endourologists in five academic institutions. Choice of procedure was at the discretion of the surgeon. The vast majority of patients underwent monopolar resection. The en-bloc procedure was performed with Collin's knife or the classic resection loop. Study end-points were surgery, catheterization and hospitalization time, presence of muscularis propria (MP) in the specimen and 3-month recurrence-free survival (RFS).

Results: The study included 427 (274 conventional TURBT vs. 153 en-bloc) patients with mean age of 69 years (range: 18-99). There were more cases with MP present in the specimen in the en-bloc group (91.3\% vs. 75.5\%; $p<0.001$ ). Surgery and hospitalization times were statistically shorter in the en-bloc group (both $p<0.05$ ). A borderline significant difference was noted when the number of residual tumours in reTURBTs was analysed, with fewer cases of residual tumour in the en-bloc group $(p=0.051)$. RFS at 3 months was higher in the en-bloc group ( $88.4 \%$ vs. $80.1 \% ; p=0.027$ ). After propensity score matching, differences in MP presence, hospitalization time and 3-month RFS status remained statistically significant.

Conclusions: When compared to conventional TURBT, en-bloc resection of bladder tumour is associated with higher percentage of MP presence in histopathological specimen, higher 3-month RFS and shorter hospitalization time.
\end{abstract}

Key words: bladder cancer, transurethral resection, transurethral resection of bladder tumour, en-bloc.

\section{Introduction}

In the vast majority of bladder cancer (BCa) cases, transurethral resection of bladder tumour (TURBT) constitutes the basis of the primary diagnosis and is the first therapeutic procedure. Because of the relatively high $\mathrm{BCa}$ incidence in the general population and because of the recurrent character of the dis-

\section{Address for correspondence}

Wojciech Krajewski MD, PhD, Department of Urology and Oncological Urology, Wroclaw Medical University, 213 Borowska St, Wrocław, Poland, phone/fax: +48 7173310 10, e-mail: wk@softstar.pl 
ease, TURBT is one of the most commonly performed urologic procedures. Despite the general conviction that TURBT is an easy procedure, the quality of its performance translates directly into further oncological results [1]. It is widely known that classic TURBT contradicts the basic principles of oncological surgery and results in scattering of numerous cancerous cells [2-4]. Additionally, antegrade loop movement precludes precise guidance of the cutting tool in the adequate bladder wall layer. Finally, because of tissue fragmentation, disorientation and thermal damage, pathologic material obtained during the resection often presents reduced diagnostic value $[5,6]$. Consequently, the en-bloc resection concept was created. During en-bloc TURBT the whole tumour is retrieved in one piece - the exophytic papillary part together with the base and bladder wall fragment. It allows for preservation of histological relations of the specimen, for increase of the rates of lamina muscularis presence and hypothetically for reduction of the cancerous cells scattering $[7,8]$.

\section{Aim}

The aim of this study was to analyse the influence of en-bloc technique on peri- and postoperative results of TURBT performed with electric current.

\section{Material and methods}

\section{Patients}

This prospective multicentre study enrolled 427 consecutive patients undergoing conventional or enbloc TURBT for both primary and recurrent, uni- and multifocal bladder tumours of 1-4 cm diameter. The bigger lesions and tumours with the visual aspect of muscle invasive disease were resected in a classical way. In the case of multiple lesions, en-bloc resection was preferably performed in all of the tumours. The inclusion criteria were as follows: age above 18 years, resection of bladder tumour, sterile urine preoperatively or ongoing directed antibiotic therapy at the time of surgery, signed informed consent. Detailed baseline patient characteristics are presented in Table I. Patients undergoing restaging resection, cold-cup biopsy, fulguration only or cystoscopy only were excluded from the analysis.

\section{Methods}

All operations were performed by five experienced endourologists in five academic institutions.
This was a non-randomized analysis - choice of procedure was at the discretion of the surgeon. Patients were blinded to the allocated arm. The vast majority of patients underwent monopolar resection. An enbloc procedure was performed with Colling's knife or a classic resection loop (Photo 1). No additional resection bed sampling was performed after resection. Some very scarce en-bloc cases requiring "conversion" to classic resection were automatically excluded from the study. A re-staging resection (reTURBT) and postoperative intravesical chemo-/immunotherapy instillations were performed according to guidelines recommendations and at the urologists' discretion based on pathologic and/or intraoperative outcomes.

The study end-points were surgery, catheterization and hospitalization time, presence of lamina muscularis propria (MP) in the specimen and 3-month recurrence-free survival (RFS).

All study data were collected by each study site in the dedicated uniform electronic form. Before the study initiation, the protocol was approved by the Institutional Review Board.

\section{Statistical analysis}

Study groups were compared using Pearson's $\chi^{2}$ test, Student's $t$-test and the Wilcoxon test. Also, owing to inherent differences between groups in terms of baseline patient and disease characteristics, randomization mimicking propensity score matching (PSM) was applied. The variables were adjusted for tumour size, focality and stage. In the reTURBT and recurrence analyses as well as in the PSM only urothelial tumours were included (excluding TO cases). Cases with TO lesions were included in operation, catheterization and hospitalization time analyses. Statistical significance was considered at $p<0.05$. Analyses were performed in the R platform $[9,10]$. The statistician was blinded to the study results.

\section{Results}

There were 274 patients treated with conventional TURBT and 153 operated on according to en-bloc technique (Table I). The groups were comparable in terms of gender, age, previous intravesical treatment and tumour focality. There were more small, recurrent and Ta tumours in the en-bloc group. Additionally, the en-bloc arm contained significantly fewer patients with TO and T2 lesions. 
Table I. Patients' baseline characteristics and comparison of groups

\begin{tabular}{|c|c|c|c|c|}
\hline Parameter & $\begin{array}{l}\text { All patients } \\
(n=427)\end{array}$ & $\begin{array}{l}\text { Conventional TURB } \\
\qquad(n=274)\end{array}$ & $\begin{array}{l}\text { En-bloc TURBT } \\
\quad(n=153)\end{array}$ & $P$-value \\
\hline Gender (M/F) & $318 / 109(74.5 / 25.5 \%)$ & 201/73 (73.4/26.6\%) & $117 / 36(76.5 / 23.5 \%)$ & 0.9 \\
\hline Age, mean \pm SD & $69 \pm 1.46$ & $69.5 \pm 11.47$ & $68 \pm 11.39$ & 0.4 \\
\hline Primary/recurrent/missing data & $\begin{array}{c}209 / 205 / 13 \\
(48.9 / 48 / 3.1 \%)\end{array}$ & $\begin{array}{c}115 / 147 / 12 \\
(42 / 53.6 / 4.4 \%)\end{array}$ & $\begin{array}{c}94 / 58 / 1 \\
(61.4 / 37.9 / 0.7 \%)\end{array}$ & $<0.001$ \\
\hline $\begin{array}{l}\text { Previous intravesical chemotherapy } \\
\text { (yes/no) }\end{array}$ & $17 / 410(4 / 96 \%)$ & $13 / 261(4.7 / 95.3 \%)$ & 4/149 (2.6/97.4\%) & 0.1 \\
\hline Previous intravesical BCG (yes/no) & $52 / 375(12.2 / 87.8 \%)$ & $39 / 235(14.2 / 85.8 \%)$ & $13 / 140(8.5 / 91.5 \%)$ & 0.1 \\
\hline Number of tumours (solitary/multiple) & $256 / 171(60 / 40 \%)$ & 159/115 (58/42\%) & $97 / 56(63.4 / 36.6 \%)$ & 0.7 \\
\hline Tumour size $(<3 \mathrm{~cm} />3 \mathrm{~cm})$ & $337 / 90(78.9 / 21.1 \%)$ & 208/66 (75.9/24.1\%) & $129 / 24(84.3 / 15.7 \%)$ & 0.003 \\
\hline \multicolumn{5}{|l|}{ Tumour stage: } \\
\hline TO & $73(17.1 \%)$ & $58(21.2 \%)$ & $15(9.8 \%)$ & 0.003 \\
\hline $\mathrm{Ta}$ & $220(51.5 \%)$ & $126(46 \%)$ & $94(61.4 \%)$ & 0.002 \\
\hline $\mathrm{T} 1$ & $96(22.5 \%)$ & $58(21.2 \%)$ & $38(24.8 \%)$ & 0.383 \\
\hline $\mathrm{T} 2$ & $38(8.9 \%)$ & $32(11.7 \%)$ & $6(3.9 \%)$ & 0.007 \\
\hline Concomitant CIS & $40(11.3 \%)$ & $19(8.8 \%)$ & $21(15.2 \%)$ & 0.063 \\
\hline \multicolumn{5}{|l|}{ Tumour grade: } \\
\hline G1 & $47(13.3 \%)$ & $21(9.7 \%)$ & $26(18.8 \%)$ & 0.014 \\
\hline G2 & $184(52 \%)$ & $113(52.3 \%)$ & $71(51.4 \%)$ & 0.9 \\
\hline G3 & $123(34.7 \%)$ & $82(38 \%)$ & $41(29.7 \%)$ & 0.1 \\
\hline $\begin{array}{l}\text { Presence of muscularis propria in the } \\
\text { specimen }\end{array}$ & $289(81.6 \%)$ & $163(75.5 \%)$ & $126(91.3 \%)$ & $<0.001$ \\
\hline $\begin{array}{l}\text { Catheterization time [h], } \\
\text { median (SD; mean) }\end{array}$ & $24(41.8 ; 37.22)$ & $24(46.82 ; 34.55)$ & $24(32.1 ; 34.6)$ & 0.6 \\
\hline $\begin{array}{l}\text { Hospitalization time [days], } \\
\text { median (SD; mean) }\end{array}$ & $1(1.6 ; 1.65)$ & $1(1.82 ; 1.89)$ & $1(0.9 ; 1.3)$ & $<0.001$ \\
\hline $\begin{array}{l}\text { Operation time [min], } \\
\text { median (SD; mean) }\end{array}$ & $30(17.6 ; 32.6)$ & $30(18.3 ; 34.55)$ & $25(16 ; 29.6)$ & 0.04 \\
\hline reTURBT & $95(26.8 \%)$ & $58(26.9 \%)$ & 37 (26.8\%) & 0.9 \\
\hline Residual tumour in reTURBT & $40(42.1 \%)$ & $29(50 \%)$ & $11(29.7 \%)$ & 0.051 \\
\hline 3-month RFS & $368(83.3 \%)$ & $231(80.1 \%)$ & $137(88.4 \%)$ & 0.027 \\
\hline
\end{tabular}

There were more cases with MP present in the histopathological specimen in the en-bloc group. The difference was strongly statistically significant.

The time of catheterisation did not differ significantly between the groups and was $22 \mathrm{~h}$ on average. On the other hand, operation and hospitalization times were significantly shorter in the en-bloc group.
Finally, tumour recurrences during the first 3 month were recorded in $16.7 \%$ of cases with fewer recurrences observed in the en-bloc group.

ReTURBT was performed in accordance with EAU guidelines in $26.8 \%$ of patients. A borderline significant difference $(p=0.051)$ was noted when the number of residual tumours in reTURBTs was anal- 

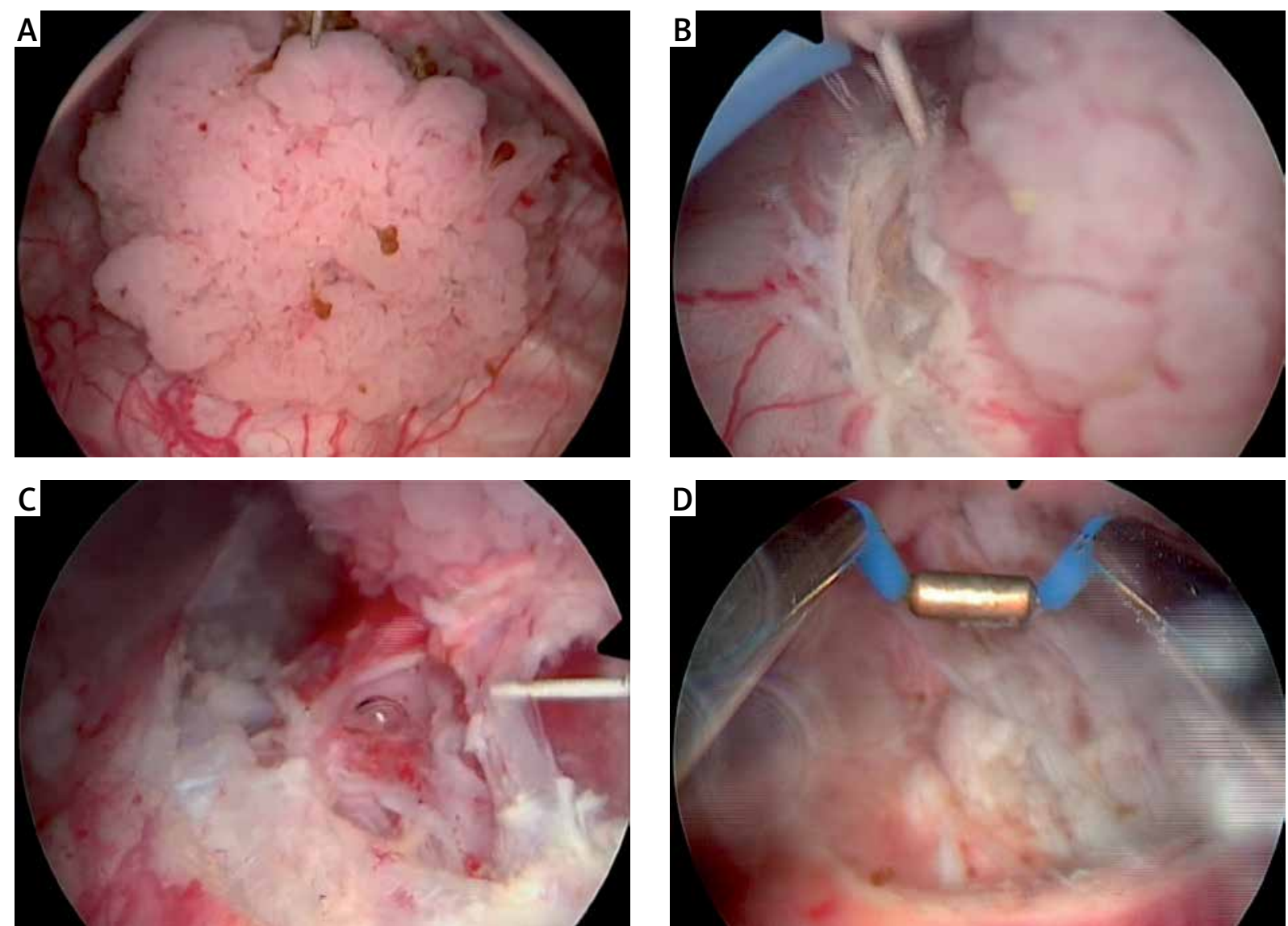

Photo 1. A - Papillary tumour, B - circumferential marking of the resection site, finding the appropriate depth of resection, $\mathbf{C}$ - mainly blunt dissection within muscular layer, $\mathbf{D}$ - resection bed

ysed, with fewer cases of residual tumour in the enbloc group. However, because of low numbers, the conclusions should be drawn with caution.

Because of the non-randomized study design and differences in baseline and tumour characteristics, PSM was implemented. Matching was performed for only urothelial tumours (excluding T0), adjusting for tumour size, focality and stage. The results of the comparison after matching are presented in Table II.

It was found that differences in detrusor muscle presences, hospitalization time and 3-month RFS remained statistically significant. On the other hand, the differences in operative time and number of re-

Table II. Group comparisons after propensity score matching

\begin{tabular}{|lccc|}
\hline Parameter & $\begin{array}{c}\text { Conventional TURBT } \\
(n=138)\end{array}$ & $\begin{array}{c}\text { En-bloc TURBT } \\
(n=138)\end{array}$ & $P$-value \\
\hline Presence of muscularis propria in the specimen & $95(68.8 \%)$ & $126(91.3 \%)$ & $<0.001$ \\
\hline Catheterization time [h], median (SD;mean) & $24(29.9 ; 33.48)$ & $24(31.9 ; 34.4)$ & 0.2 \\
\hline Hospitalization time [days], median (SD;mean) & $1(1.13 ; 1.54)$ & $1(0.87 ; 1.27)$ & 0.002 \\
\hline Operation time [min], median (SD;mean) & $30(16.2 ; 32.44)$ & $25(16.13 ; 29.4)$ & 0.059 \\
\hline reTURBT & $30(21.7 \%)$ & $37(26.8 \%)$ & 0.3 \\
\hline Residual tumour in reTURBT & $17(12.3 \%)$ & $13(9.4 \%)$ & 0.5 \\
\hline 3-month RFS & $101(73.2 \%)$ & $122(88.4 \%)$ & 0.001 \\
\hline
\end{tabular}

SD - standard deviation, TURBT - transurethral resection of the bladder tumour. The value of adjusted $p<0.05$ was considered statistically significant (bolded). 
sidual tumours in reTURB did not reach statistical significance.

\section{Discussion}

There is growing evidence that en-bloc resection improves the results of the resection and the quality of the histopathological specimen.

In this population, after performing classic comparison of the study groups, it was demonstrated that en-bloc resection of bladder tumour was associated with a higher percentage of MP presence in the histopathological specimen, a lower rate of residual tumour in reTURB, a lower rate of recurrence at 3 months and shorter hospitalization time. Also, the operation time was shorter.

As en-bloc is a very precise operation based on blunt dissection within the muscular layer using limited energy mainly in "flash-firing" fashion, it is clear that the amount and quality of the muscle in the specimen is in the majority of cases sufficient to allow correct histopathological assessment [7, 8]. It is worth highlighting that the muscular layer absence in the specimen is associated with a significant risk of residual disease and early recurrence [6]. This, together with avoidance of tumour fragmentation and implementation of a more methodical operation, may have caused us to find fewer residual tumours in reTURBT and fewer recurrences during 3 months. On the other hand, it might be caused by the fact that the en-bloc group contained more lower risk tumours - lesions in this arm were smaller, less advanced and better differentiated. Smaller tumours in the en-bloc group clearly represent the qualification criteria and are an obvious limitation of the non-randomized character of this study. The tumour must finally fit and be pulled out in one piece through the working channel of the endoscope. This may be challenging in bigger and/or more cohesive tumours, and therefore, en-bloc resection is preferably performed in the case of a smaller lesion. As a result, the procedure takes less time. A possible explanation for the differences in pathological stage and/or grade may be once more the non-randomized design and the general tendency to resect in one piece typical, papillary tumours, that do not exceed a certain size rather than non-characteristic, sessile lesions that finally, in many cases, turned out to be benign or aggressive and invasive.

Because of the clear limitations resulting from non-randomisation, we performed propensity score matching with further analysis adjusting for tumour size, focality and stage. Interestingly, the differences in detrusor muscle presences, hospitalization time and 3 months recurrence status remained strongly statistically significant. However, the borderline differences in operative time and number of residual tumours in reTURBTs did not reach statistical significance after PSM.

In the available literature various studies presenting numerous electric tools for en-bloc resection may be found. En-bloc TURBT may be performed by means of a classic resection loop, but also with the J-electrode, Colling's knife, a flat loop, or a bipolar button [9-15]. Additionally, several studies comparing electric en-bloc resection and classical TURB have been published. In the study by Zhang et al., the authors compared the results of resection performed in 90 patients and determined that the muscle layer could be found in $100 \%$ of en-bloc specimens, but only in $70 \%$ of classic TURB samples. On the other hand, they did not find significant differences in operation time, rate of surgical complications or recurrence incidence between the two study groups [15]. In the prospective analysis by Sureka et al. on only 45 patients the authors found that en-bloc resection was associated with a significant reduction in the recurrence rate, but not with the progression rate [14]. Finally, in the prospective study by Balan et al. 90 patients were randomized to undergo bipolar button en-bloc or classic, monopolar TURB. A reduced catheterization time and hospital period, as well as lower recurrence rate, were found in the en-bloc group [13]. There is no metanalysis in the available literature comparing conventional TURBT with en-bloc performed with electric tools. In the metanalysis by Wu et al. the authors compared conventional TURBT with laser en-bloc resections and concluded that en-bloc was associated with shorter hospitalization and catheterization times and lower complication and recurrence-free rates, and provided specimens of better quality [16].

Despite several strengths, our study is not free from limitations. First and foremost, this study is limited by its non-randomized nature, and thus burdened with particular flaws mentioned above. On the other hand, the size of our cohort and the use of propensity match analyses partially reduce the selection bias. Second, no standardized pathologic review was performed, although every centre benefits from a pathologist specializing in uropathology. Also, nei- 
ther variant histologies nor lymphovascular invasion were analysed; however, their possible influence on the study results would be rather small. Third, no progression analysis was performed. Fourth, no data regarding the tumour location were available in our database, so no definitive conclusion can be made regarding this aspect. Fifth, no analysis of surgeon volume was included. However, all of the surgeons are highly experienced in endourology. Finally, the follow-up was too short to draw conclusions regarding long-term oncological outcomes.

\section{Conclusions}

This study showed that, compared to conventional TURBT, en-bloc TURBT is associated with a higher percentage of MP in the histopathological specimen, higher 3-month RFS and shorter hospitalization time.

\section{Conflict of interest}

The authors declare no conflict of interest.

\section{References}

1. Brausi M, Collette L, Kurth K, et al. Variability in the recurrence rate at first follow-up cystoscopy after TUR in stage Ta T1 transitional cell carcinoma of the bladder: a combined analysis of seven EORTC studies. Eur Urol 2002; 41: 523-31.

2. Zhou Z, Zhao S, Lu Y, et al. Meta-analysis of efficacy and safety of continuous saline bladder irrigation compared with intravesical chemotherapy after transurethral resection of bladder tumors. World J Urol 2019; 37: 1075-84.

3. Sylvester RJ, van der Meijden AP, Oosterlinck W, et al. Predicting recurrence and progression in individual patients with stage Ta T1 bladder cancer using EORTC risk tables: a combined analysis of 2596 patients from seven EORTC trials. Eur Urol 2006; 49: 466-5.

4. Engilbertsson H, Aaltonen KE, Bjornsson S, et al. Transurethral bladder tumor resection can cause seeding of cancer cells into the bloodstream. J Urol 2015; 193: 53-7.

5. Naselli A, Puppo P. En bloc transurethral resection of bladder tumors: a new standard? J Endourol 2017; 31: S20-S4.

6. Gontero P, Sylvester R, Pisano F, et al. Prognostic factors and risk groups in T1G3 non-muscle-invasive bladder cancer patients initially treated with Bacillus Calmette-Guerin: results of a retrospective multicenter study of 2451 patients. Eur Urol 2015; 67: 74-82.

7. Kramer MW, Altieri V, Hurle R, et al. Current evidence of trans urethral en-bloc resection of nonmuscle invasive bladder cancer. Eur Urol Focus 2017; 3: 567-76.

8. Kramer MW, Rassweiler JJ, Klein J, et al. En bloc resection of urothelium carcinoma of the bladder (EBRUC): a European multicenter study to compare safety, efficacy, and outcome of laser and electrical en bloc transurethral resection of bladder tumor. World J Urol 2015; 33: 1937-43.

9. Saito S. Transurethral en bloc resection of bladder tumors. J Urol 2001; 166: 2148-50.

10. Lodde M, Lusuardi L, Palermo S, et al. En bloc transurethral resection of bladder tumors: use and limits. Urology 2003; 62: 1089-91.

11. Hurle R, Lazzeri M, Colombo P, et al. "En bloc" resection of nonmuscle invasive bladder cancer: a prospective single-center study. Urology 2016; 90: 126-30.

12. Zhang J, Wang L, Mao S, et al. Transurethral en bloc resection with bipolar button electrode for non-muscle invasive bladder cancer. Int Urol Nephrol 2018; 50: 619-23.

13. Balan GX, Geavlete PA, Georgescu DA, et al. Bipolar en bloc tumor resection versus standard monopolar TURBT - which is the best way to go in non-invasive bladder cancer? Rom J Morphol Embryol 2018; 59: 773-80.

14. Sureka SK, Agarwal V, Agnihotri S, et al. Is en-bloc transurethral resection of bladder tumor for non-muscle invasive bladder carcinoma better than conventional technique in terms of recurrence and progression? A prospective study. Indian J Urol 2014; 30: 144-9.

15. Zhang KY, Xing JC, Li W, et al. A novel transurethral resection technique for superficial bladder tumor: retrograde en bloc resection. World I Surg Oncol 2017; 15: 125.

16. Wu YP, Lin TT, Chen SH, et al. Comparison of the efficacy and feasibility of en bloc transurethral resection of bladder tumor versus conventional transurethral resection of bladder tumor: a meta-analysis. Medicine (Baltimore) 2016; 95: e5372.

Received: 25.01.2020, accepted: 2.03.2020. 